\title{
Towards Routine EDX Tomography in Semiconductor Failure Analysis
}

\author{
Frieder H. Baumann ${ }^{1 *}$, Brian Popielarski ${ }^{1}$, Travis Mitchell ${ }^{1}$ and Yinggang $\mathrm{Lu}^{2}$ \\ 1. Globalfoundries, Malta, NY, USA. \\ 2. Thermofisher Scientific, Hillsboro, OR, USA. \\ *Corresponding author: Frieder.Baumann@Globalfoundries.com
}

Modern Very Large Scale Integrated (VLSI) circuits consist of devices which are inherently 3D in nature (tri-gate transistors, fin-FETs, NAND memory cells) and which approach nanometer dimensions. Characterization of these devices and their defects requires chemically sensitive 3D techniques with near atomic resolution. Chemically sensitive electron tomography using EDX (electron dispersive X-ray) [1] [2] or EELS (electron energy loss spectroscopy) [3] has been demonstrated to be able to successfully deliver 3D information of modern electronic devices with nanometer resolution.

There are, however, several reasons why EDX tomography has not been employed routinely in semiconductor failure analysis, one reason being the fact that data acquisition of an EDX tomography is highly time consuming, blocking a tool for many hours. Another reason is the low x-ray count and detector shadowing by the sample holder, leading to possible artifacts in the reconstruction [4].

In this paper, we show how a programmable CD-TEM (Critical Dimension TEM) can be used to run fully autonomously a script (recipe) to collect the EDX maps at all the required angles. The tool can be programmed to run autonomously during off-hours (night-shifts, sparsely populated shifts); only sample loading and recipe start need user interaction. We also demonstrate how a modified sample holder/grid greatly increases both the total Xray counts and its homogeneity versus tilt angle.

For data acquisition, a commercially available, programmable TEM tool equipped with four windowless SDD detectors was used, operating at 80 or $200 \mathrm{kV}$. The instrument is equipped with a programming environment, which consists of a multitude of commands (e. g., image shift, auto focus, acquire image, acquire EDS map, etc), which can be stitched together to obtain a fully functioning recipe. In addition, exception handlers allow the user to create very robust, crash-resistant scripts which are designed to run autonomously without user interaction.

To minimize possible shadowing effects by a specimen holder, an on-axis rotational tomography holder was used for our approach. Originally, this holder accepts cone or rod-like specimens mounted on a post. This post is then inserted in the holder. Instead of a post, a clamp mechanism was constructed and built, which allows the mounting of a TEM grid (fig. 1). After holder insertion, the TEM grid is exposed free as a cantilever in the objective lens, thus eliminating any shadowing by the specimen holder. To maximize the X-ray yield, the post of the TEM grid was bevel cut in a focused ion beam tool (fig. 2). Figure 3 shows the comparison between the cantilevered holder and a commercially available low background tomography holder. The cantilevered holder shows dramatically less shadowing for three of the four detectors, and was used in the following examples: Figure 4 shows the reconstruction of a source/drain defect in a modern FinFET circuit. The data set consisted of 27 maps collected at $200 \mathrm{kV}$. Figure 5 shows a failing contact structure in an experimental FinFET design; 31 maps were acquired at $80 \mathrm{kV}$ for 5 minute each.

The steps described above allow one to routinely acquire time consuming EDX tomograms in off-hours by running an analytical TEM fully automatically and autonomously. Displaying the full 3D distribution of the elements comprising the device can be a powerful tool in understanding complex failure mechanisms in modern electronic circuits.

References:

[1] A Genç et al., Microscopy and Analysis 116, p. 23.

[2] K Lepinay et al., Micron. 47 (2013), p. 43. 
[3] FH Baumann et al., Microsc. Microanal. 22 (S3) (2016).

[4] TJA Slater et al., Ultramicroscopy 162 (2016), p. 61.

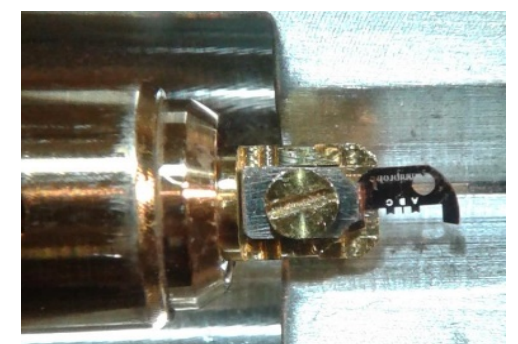

Figure 1. Tip of tomography sample holder (left), showing the clamp with screw (middle), which holds the TEM grid.

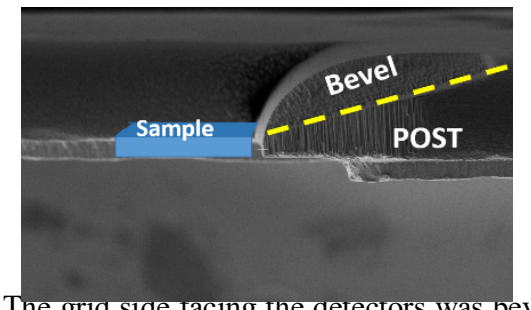

Figure 2. The grid side tacing the detectors was bevel cut at an angle of about $20^{\circ}$. That reduces the shadowing of the detectors to a minimum (sample cartoon not to scale).
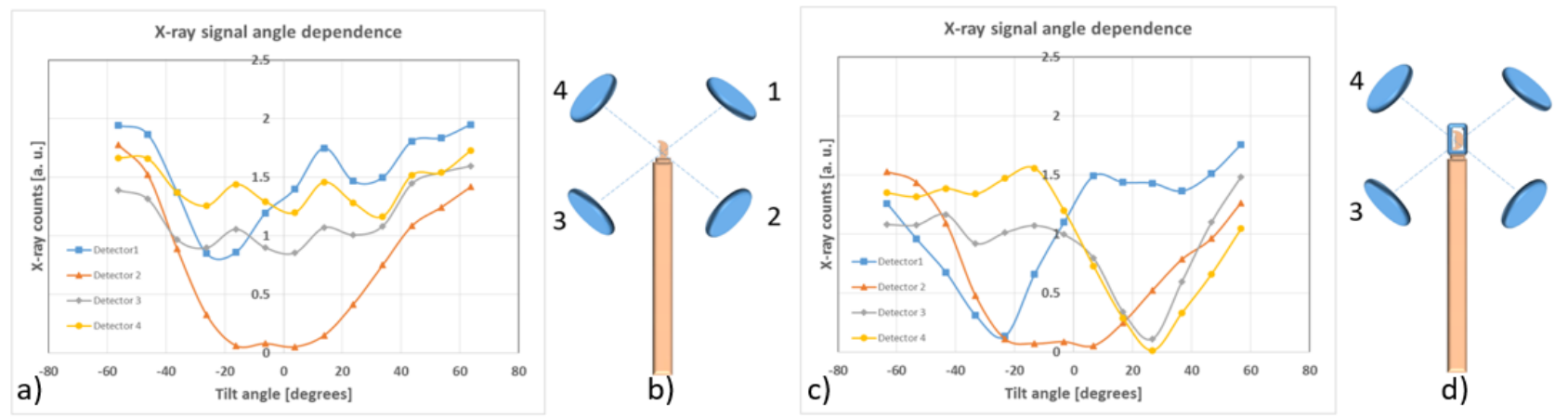

Figure 3. Measured X-ray counts vs tilt angle for a homogenous Si film. For the cantilevered holder (b), only detector 2 shows significant shadowing of the X-rays (a). When measuring the same sample in a commercial low-background analytical tomography holder (d), all four detectors show strong shadowing for a range of angles (c) due to the presence of the holder.

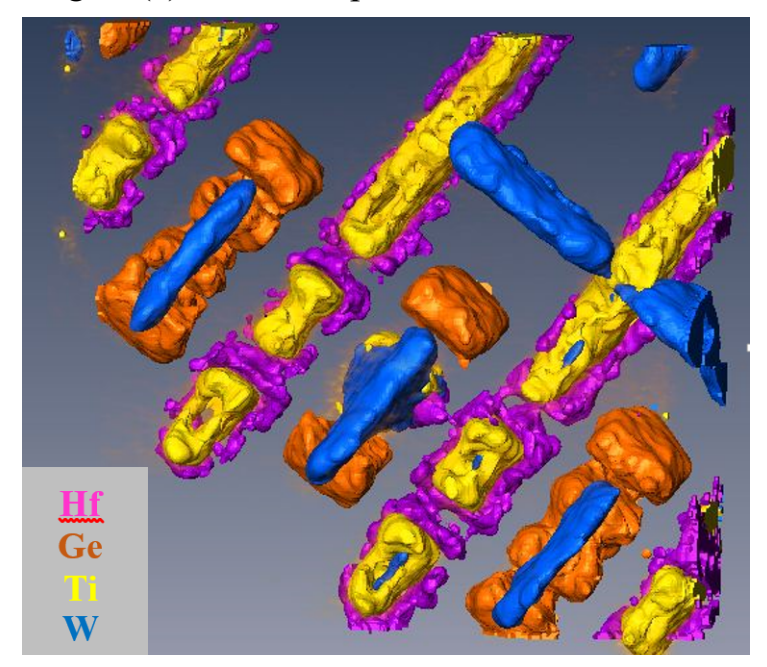

Figure 4. 3D reconstruction of a modern FinFET structure. The gates (TiN and Hf) extend from top right to bottom left (the Si fins are not displayed). The total field of view is roughly $250 \mathrm{~nm}$, the resolution of the 3D image is about $1 \mathrm{~nm}$.

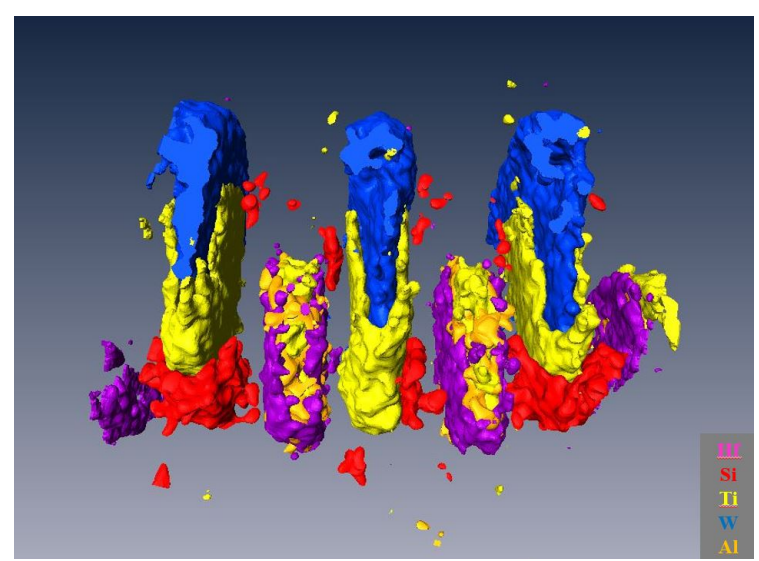

Figure 5. 3D elemental mapping of a similar FinFET structure. The central W+Ti contact has missing epitaxial $\mathrm{Si}$, resulting in a short due to $\mathrm{Ti}$ overfill. This is easy to see in the tomographic reconstruction, but almost impossible to discern as contrast in normal TEM or STEM image. 